\title{
Erratum to: Polypropylene Mesh for Pelvic Organ Prolapse Surgery
}

\author{
Nir Haya $\cdot$ Christopher Maher
}

Published online: 5 October 2013

(C) Springer Science+Business Media New York 2013

Erratum to: Curr Obstet Gynecol Rep (2013) 2:129-138

DOI 10.1007/s13669-013-0045-8

The original version of this article contained author query prompts (i.e. "AU Query") within the text on page 130 (" $\{$ AU Query: Is [2] the correct reference citation?\}") and 135 ("\{AU Query: As meant: the United States?\}”). These were not to be included within the final published manuscript.

The online version of the original article can be found at http://dx.doi.org/ 10.1007/s13669-013-0045-8.

N. Haya $\cdot$ C. Maher

Royal Brisbane and Women's Hospital, Suite 8630 Chasely Street,

Auchenflower,

Brisbane 4066, Australia

N. Haya $\cdot$ C. Maher $(\bowtie)$

Wesley Urogynaecology, Suite 8630 Chasely Street,

Auchenflower,

Brisbane 4066, Australia

e-mail: chrismaher@urogynaecolgy.com.au

C. Maher

University of Queensland, Suite 8630 Chasely Street,

Auchenflower,

Brisbane 4066, Australia 